\title{
ANÁLISE DA VOLATILIDADE DE PREÇOS DE PRODUTOS AGROPECUÁRIOS NO BRASIL ${ }^{1}$
}

\author{
Kilmer Coelho Campos ${ }^{2}$
}

\begin{abstract}
Resumo - A instabilidade de renda dos produtores e investidores, proveniente de flutuações nos preços, representa um problema cujas características e causas devem ser amplamente pesquisadas, em vista da importância da commodity no agronegócio nacional e das perdas que essas flutuações causam na lucratividade, nos empregos e nas divisas para o Brasil. Isto posto, utiliza-se a classe de modelos de heterocedasticidade condicional auto-regressiva (ARCH e GARCH), para caracterizar e analisar a volatilidade das séries de retornos mensais da soja, café, milho e boi gordo. A análise empírica da volatilidade mostra que esses produtos são marcados por acentuadas flutuações nos preços, em que choques positivos ou negativos geram impactos com período longo de duração. No somatório dos coeficientes de reação e persistência da volatilidade obtiveram-se valores próximos ou maiores do que um, o que indica que os choques na volatilidade irão perdurar por algum tempo.
\end{abstract}

Palavras- chave: volatilidade de preços, produtos agropecuários, Brasil.

\section{Introdução}

Segundo Baccarin apud Pimentel, Almeida e Sabbadini (2004), no final da década de 80 e começo da década de 90 , houve alteração do quadro da política comercial brasileira, caracterizando-se pelo que seria chamada a abertura comercial brasileira, em que, por meio da redução nas barreiras alfandegárias de ordem tarifária, o país se abria às importações e permitia à sua indústria concorrer com os produtos elaborados no exterior.

Outras medidas importantes, neste período, também contribuíram para o desempenho exportador do Brasil, como a implementação do Mercado Comum do Sul (Mercosul) e a reforma monetária no país, responsável

Recebido em: 25/05/2007 Aceito em: 15/08/2007

2 Doutorando em Economia Aplicada pelo Departamento de Economia Rural da UFV.

E-mail: kilmercc@bol.com.br 
por um longo período de sobrevalorização da moeda nacional e posterior desvalorização cambial, que, além de engendrar volatilidade às expectativas comerciais, afetou o desempenho exportador nacional, sobretudo as exportações agrícolas, na medida em que fizeram variar os preços percebidos pelos produtores nacionais, bem como pelos consumidores externos.

Em decorrência desses fatores, nos últimos anos a agropecuária brasileira vem se desenvolvendo e modernizando, destacando-se como atividade de alto valor econômico e social, com tendência de crescimento acentuado no curto prazo. A amplitude dos mercados interno e externo contribuiu para o caráter dinâmico deste setor no Brasil, com a incorporação de modernas tecnologias produtivas para satisfazer às exigências desses mercados.

Melhor desempenho competitivo pode ser resultado da competitividade de preços, explicada, primeiramente, pela opção dos importadores por escolherem diferentes exportadores de produtos e serviços, ou seja, os importadores tendem a substituir o consumo de bens, cujos preços se elevaram, pelo consumo dos que apresentaram redução no preço, em termos relativos (Oranje, 2003).

Portanto, a análise de preços e de suas oscilações é um dos principais instrumentos para planejamento e avaliação de atividades agropecuárias, visto que é fator decisivo na escolha das oportunidades empresariais. A formação do preço, como elemento controlador do mecanismo de troca, reveste-se de singular importância para o Governo na formulação e na aplicação de políticas eficientemente direcionadas ao setor agropecuário. A geração e a adoção de tecnologia por parte do produtor têm como incentivo a rentabilidade esperada, e as relações de preço dos insumos e a análise de preço do produto são elementos básicos na tomada de decisões.

As flutuações cíclicas e, ou, sazonais dos preços dos produtos agropecuários provocam instabilidade tanto na renda do produtor como nas despesas dos consumidores urbanos. Essa instabilidade pode provocar 
desestímulos à produção, em períodos de baixa dos preços, ou excesso de produção, em períodos de preços muito elevados. Assim, é necessário conhecer o padrão de flutuação sazonal ou a volatilidade desses preços, para que possam ser implementadas políticas de estabilização dos preços dos produtos agropecuários, ao longo do ano.

Segundo Rodrigues (2001), a análise do comportamento de séries históricas de preços é de fundamental importância para a economia, visto que praticamente todas as fases das relações econômicas estão diretamente relacionadas com preços. A análise dos preços agropecuários se configura como ponto de interesse geral, dada a ampla gama de interrelações com outros setores de atividade e agentes econômicos, principalmente em uma economia com vocação agropecuária como a do Brasil, em que as atividades ligadas ao agronegócio são responsáveis por, aproximadamente, $32 \%$ do PIB nacional.

Ressalta-se a importância desse tipo de análise no processo de tomada de decisão sob situação de incerteza, que incide sobre os agentes econômicos em diversos ramos de atividade e, particularmente, sobre os envolvidos nos mercados financeiros e de commodities (soja, café, milho, boi gordo, etc). Tanto os hedgers, que estão envolvidos em transações físicas de produtos, querem proteger-se de futuras oscilações de preços, quanto os especuladores, que querem assumir riscos nesses mercados em razão de possíveis ganhos futuros, necessitam de informações sobre variáveis e ativos que são fundamentais para a rentabilidade de suas atividades (Schwager, 1995).

Logo, a oscilação na renda de produtores e investidores, proveniente de flutuações nos preços agropecuários, configura-se como um problema cujas características e causas devem ser amplamente pesquisadas, dada a importância da commodity para o agronegócio nacional e dadas as perdas que essas flutuações podem provocar tanto na lucratividade para o setor quanto nos empregos e divisas para o Brasil. 
Dessa forma, neste artigo utiliza-se a classe de modelos de heterocedasticidade condicional auto-regressiva (ARCH e GARCH), para caracterizar e analisar a volatilidade das séries de retornos mensais da soja, café, milho e boi gordo. Pretende-se, assim, fornecer subsídios ao delineamento de estratégias adequadas ao gerenciamento do risco de variações nos preços (retornos) dessas commodities.

Na seção 2 deste trabalho, faz-se uma breve revisão do marco conceitual analítico, relevante para ilustrar a importância da classe de modelos ARCH e GARCH na análise da volatilidade de séries de preços de produtos agropecuários; na seção 3, expõe-se a fonte dos dados utilizados na análise; na seção 4 , procede-se à apresentação dos resultados e à discussão. Finalmente, na seção 5, são postas as conclusões do estudo aplicado.

\section{Referencial analítico}

Ao analisar as séries temporais, podem-se utilizar dois enfoques básicos, com o objetivo de elaborar modelos para as séries com propósitos determinados. O primeiro enfoque aborda a análise no domínio temporal com modelos paramétricos propostos e o segundo, a análise baseada no domínio de frequiências. Os modelos propostos são não-paramétricos.

A partir de uma série temporal observada em intervalos de tempo, podese investigar o mecanismo gerador da série temporal, fazer previsões de valores futuros da série, descrever apenas o comportamento da série e procurar periodicidades relevantes dos dados.

Os procedimentos de previsão de séries temporais indicam que séries, principalmente financeiras, como preços de ações, taxas de inflação, taxas de câmbio, dentre outras, apresentam valores que oscilam, consideravelmente, de um período para outro. Observou-se que os erros de previsão são relativamente pequenos em alguns períodos e relativamente grandes em outros. Tal variabilidade pode ser explicada 
pela volatilidade no mercado financeiro, em decorrência de mudanças nas políticas monetária e fiscal do governo e nas relações internacionais de comercialização de produtos. Isto prova que a variância dos erros de previsão não é constante, mas varia de um período para outro, ou seja, há uma espécie de autocorrelação na variância dos erros de previsão (Gujarati, 2000).

Supondo que o comportamento dos erros de previsão depende do comportamento das perturbações da regressão, pode-se apresentar uma justificativa para a autocorrelação na variância das perturbações. Assim, para capturar esta correlação, Engle (1982) desenvolveu o modelo autoregressivo de heterocedasticidade condicional $(\mathrm{ARCH})$.

Os modelos ARCH, ou modelos auto-regressivos com heterocedasticidade condicional, foram introduzidos por Engle (1982), com o objetivo de estimar a variância da inflação. A idéia básica é que o retorno $\mathrm{Y}_{\mathrm{t}}$ é não-correlacionado serialmente, mas a volatilidade (variância condicional) depende de retornos passados por meio de uma função quadrática (Morettin; Toloi, 2004).

A idéia principal do modelo ARCH é o fato de que a variância de " $\varepsilon$ ", no período de tempo t, depende do tamanho do quadrado do termo de erro no período $\mathrm{t}-1$, ou seja, depende de $\varepsilon_{\mathrm{t}-1}^{2}$. O termo de erro $\varepsilon_{\mathrm{t}}$, condicionado à informação disponível no período $(\mathrm{t}-1)$, seria distribuído conforme a seguinte notação:

$\varepsilon_{\mathrm{t}} \sim \mathrm{N}\left[0,\left(\alpha_{0}+\mathrm{a}_{1} \varepsilon_{\mathrm{t}-1}^{2}\right)\right]$, em que $\alpha_{0}$ e $\alpha_{1}$ são parâmetros explicativos da variância do termo de erro " $\varepsilon_{\mathrm{t}}$ ".

Logo, a variância de " $\varepsilon$ ", no período t, dependerá de um termo constante mais o quadrado do erro no período t-1. Esse seria o chamado processo ARCH (1), que pode ser generalizado para "r" defasagens de $\varepsilon^{2}$. As restrições paramétricas $\alpha_{0}>0, \alpha_{i}>0$, para todo $i=2 \ldots$ p e $\sum \alpha_{i}<1$, são necessárias para assegurar que a variância condicional seja positiva e fracamente estacionária. As inovações, representadas por $\varepsilon_{\mathrm{t}}$, são nãocorrelacionadas serialmente e não-estocasticamente independentes, haja 
vista que são relacionadas em seus segundos momentos (Lamounier, 2001).

Um modelo ARCH (r) pode ser definido por:

$\mathrm{Y}_{\mathrm{t}}=\beta_{0}+\beta_{1} \mathrm{X}_{1 \mathrm{t}}+\ldots+\beta_{\mathrm{k}} \mathrm{X}_{\mathrm{kt}}+\stackrel{\circ}{\mathrm{a}}_{\mathrm{t}}$

$\operatorname{Var}(\underset{\mathrm{a}}{\mathrm{a}})=\sigma_{\mathrm{t}}^{2}=\alpha_{0}+\alpha_{1} \mathrm{Y}_{\mathrm{t}-1}^{2}+\alpha_{2} \mathrm{Y}_{\mathrm{t}-2}^{2}+\ldots+\alpha_{\mathrm{r}} \mathrm{Y}_{\mathrm{t}-\mathrm{r},}^{2}$

em que $\left(\varepsilon_{t}\right)$ é uma seqüência de variáveis aleatórias independentes e identicamente distribuídas (i.i.d.), com média zero e variância unitária, $\alpha_{0}>0, \alpha_{\mathrm{i}} \mathrm{e}^{\prime \prime} 0, \mathrm{i}>0$. Na prática, supõe-se, usualmente, $\varepsilon_{\mathrm{t}} \sim \mathrm{N}(0,1)$ ou $\varepsilon_{\mathrm{t}}$ $\sim \mathrm{t}_{\mathrm{v}}(\mathrm{t}$, de Student, com v graus de liberdade).

O modelo proposto por Engle (1982) pode ser descrito pela distribuição dos erros de um modelo auto-regressivo linear dinâmico. Dado que $\mathrm{P}_{\mathrm{t}}$ é o preço de um ativo no instante $t$, então a variação de preços entre os instantes ${ }_{\mathrm{t}-1} \mathrm{e}_{\mathrm{t}}$ é dada por $\Delta \mathrm{P}_{\mathrm{t}}=\mathrm{P}_{\mathrm{t}}-\mathrm{P}_{\mathrm{t}-1}$. Denotando $\mathrm{p}_{\mathrm{t}}=\log \mathrm{P}_{\mathrm{t}}$ (sendo o logaritmo na base e), define-se o retorno composto continuamente ou $\log$-retorno por $\mathrm{r}_{\mathrm{t}}=\log \left(\mathrm{P}_{\mathrm{t}}\right)-\log \left(\mathrm{P}_{\mathrm{t}-1}\right)$, ou seja, toma-se o logaritmo dos preços e depois a primeira diferença (Moretti; Toloi, 2004).

As séries econômicas e financeiras apresentam características comuns às demais séries temporais, como tendências, sazonalidade, pontos influentes (atípicos), heterocedasticidade condicional e não-linearidade. Já os retornos financeiros apresentam características que muitas séries não possuem, como, por exemplo, os retornos, que raramente mostram tendências ou sazonalidades, com exceção eventual de retornos intradiários (Morettin; Toloi, 2004).

De acordo com os autores citados, os retornos são, em geral, nãoautocorrelacionados; os quadrados dos retornos são autocorrelacionados, o que mostra uma correlação de defasagem pequena e depois uma queda lenta nas demais correlações; as séries de retornos retratam agrupamentos de volatilidades ao longo do tempo; a distribuição (incondicional) dos retornos apresenta caudas mais pesadas e com mais 
observações do que o normal, nos extremos das caudas, do que numa distribuição normal; e algumas séries de retornos são não-lineares.

Segundo Bollerslev (1986), uma generalização do modelo ARCH pode ampliar o conjunto de informações apresentado pela série temporal e obter uma formulação mais parcimoniosa, com vistas em apresentar menos parâmetros do que um modelo AR ou MA puro. Portanto, o modelo GARCH, para a variância condicional, pode ser utilizado na descrição da volatilidade com menos parâmetros do que um modelo ARCH.

Segundo Lamounier (2001), para o modelo GARCH de ordem $(1,1)$, tem-se que a variância dos erros de um modelo econométrico ou de séries temporais, no período $t$, dependerá basicamente de três termos, ou seja, de um termo médio ou constante $\omega$; de inovações (choques) na volatilidade, que é determinada pelo quadrado dos resíduos $\left(\varepsilon_{t-1}^{2}\right)$ do período t-1, representado pelo termo $\mathrm{ARCH}$ (informações defasadas da volatilidade); e da revisão da volatilidade feita no último período $\left(\sigma_{\mathrm{t}-1}^{2}\right)$, que é o termo GARCH (variâncias previstas passadas).

Assim, o modelo GARCH $(1,1)$ pode ser representado pela notação abaixo:

$\mathrm{Y}_{\mathrm{t}}=\beta_{0}+\beta_{1} \mathrm{X}_{1 \mathrm{t}}+\ldots+\mathrm{X}_{\mathrm{kt}}+\varepsilon_{\mathrm{t}}$

$\operatorname{Var}(\varepsilon)=\sigma_{t}^{2}=\omega+\alpha \mathrm{Y}_{\mathrm{t}-1}^{2}+\beta_{1} \sigma_{\mathrm{t}-1}^{2}$.

No modelo GARCH (r,m), ou seja, modelo auto-regressivo com heterocedasticidade condicional generalizada, tem-se " $r$ " representado pela ordem do componente ARCH e "m", pela ordem do componente GARCH, sendo generalizado e representado por:

$$
\sigma_{\mathrm{t}}^{2}=\omega+\alpha \mathrm{Y}_{\mathrm{t}-1}^{2}+\alpha_{2} \mathrm{Y}_{\mathrm{t}-2}^{2}+\ldots+\alpha_{\mathrm{r}} \mathrm{Y}_{\mathrm{t}-\mathrm{r}}^{2}+\beta_{1} \sigma_{\mathrm{t}-1}^{2}+\beta_{2} \sigma_{\mathrm{t}-2}^{2}+\ldots+\beta_{\mathrm{m}} \sigma_{\mathrm{t}-\mathrm{m}}^{2} .
$$

Bollerslev et al. (1994) verificaram que a especificação mais robusta, verificada nas aplicações, é a do modelo $\operatorname{GARCH}(1,1)$, pois esta classe do modelo apresenta poucas restrições nos parâmetros. As condições 
impostas para que a variância do processo seja positiva e fracamente estacionária são w, $\alpha_{1}>0 ; \beta_{1}>0$ e $\alpha_{1}+\beta_{1}<1$. A partir dessas implicações, pode-se afirmar que a persistência de choques na volatilidade da série de retornos é medida pela soma de $\alpha_{1}$ e $\beta_{1}$.

Então, o somatório dos coeficientes que apresentam valores baixos (próximos de zero) indica que um choque inicial sobre a volatilidade irá provocar efeitos rápidos sobre o comportamento das séries e que, após curto período de tempo, a variância da série deverá convergir à sua média histórica. Entretanto, quanto maior (mais próximo de um) for o valor do coeficiente de persistência, mais vagarosamente o choque sobre a volatilidade irá se dissipar e, portanto, maior será a demora do processo de reversão à média para a variância. Se o valor do coeficiente de persistência for maior ou igual a um, os choques na volatilidade irão perdurar por um período extremamente longo na série. Isto posto, diz-se que a variância condicional de " $\varepsilon_{\mathrm{t}}$ " terá raiz unitária e a variância permanecerá elevada, não apresentando reversão à sua média histórica. Contudo, isso não implica que a série de resíduos " $\varepsilon_{t}$ " não seja estacionária, mas sim que a variância incondicional de " $\varepsilon_{\mathrm{t}}$ " não o será (Lamounier, 2001).

Observam-se, nos modelos GARCH, as mesmas vantagens e desvantagens dos modelos ARCH. Assim, volatilidades altas são precedidas de retornos ou volatilidades grandes, observando-se grupos de volatilidades presentes em séries financeiras. A identificação da ordem de um modelo GARCH, a ser ajustado para uma série, é usualmente difícil. Portanto, devem-se usar modelos de ordem baixa e escolher o melhor com base em critérios como o AIC ou BIC, de acordo com valores assumidos pela assimetria e curtose, valores da log-verossimilhança e de alguma função perda (Morettin; Toloi, 2004).

A estimação dos parâmetros dos modelos ARCH e GARCH é feita pelo método de máxima verossimilhança condicional, após adotar-se uma distribuição paramétrica para as inovações. A função de máxima verossimilhança é maximizada por métodos numéricos, sujeita às restrições de negatividade necessárias. 
Neste trabalho utilizou-se o método de Marquardt (1963). Para identificar a presença de heterocedasticidade condicional auto-regressiva, aplicouse o teste do tipo multiplicador de Lagrange proposto por Engle (1982), em que a estatística de teste tinha distribuição qui-quadrada. Logo, compara-se o valor calculado com o tabelado, para testar a hipótese nula de não-evidência de heterocedasticidade condicional.

\section{Fonte dos dados}

Utilizaram-se dados secundários correspondentes às séries de preços médios mensais recebidos pelos produtores brasileiros. As séries abrangem os períodos de janeiro de 1967 a julho de 2006, com preços de soja $(\mathrm{R} \$ / 60 \mathrm{~kg})$, café em coco $(\mathrm{R} \$ / 60 \mathrm{~kg})$, milho $(\mathrm{R} \$ / 60 \mathrm{~kg})$ e boi gordo para corte $(\mathrm{R} \$ / \mathrm{kg})$, perfazendo um total de 475 observações. Utilizaramse os dados de 1967 a 2006, com a finalidade de abranger maior período de análise do comportamento de preços dos produtos agropecuários e com a intenção de evitar a análise de estimativas, de curto e, ou, médio prazos, de resultados de séries temporais que apresentam, em sua grande maioria, fortes tendências (movimentos ascendentes ou descendentes continuados) que distorcem a verdadeira interpretação dos fatos econômicos.

Os dados foram obtidos da Fundação Getúlio Vargas - Preços Agropecuários (FGVDADOS), tendo a série de preços dos produtos sido convertida para valores atualizados pelo índice de preços recebidos (IPR) pelos produtores, utilizando-se o fator de multiplicação para deflacionar as séries de preços, na data de outubro de 2006.

Conforme Morettin e Toloi (2004), um dos objetivos em finanças é avaliar riscos de carteira de ativos financeiros, e o risco é constantemente medido pelas variações de preços dos ativos. Portanto, a variação relativa de preços entre instantes de tempo gera os retornos, que são livres de escala e apresentam propriedades estatísticas mais interessantes, como estacionariedade e ergodicidade. Uma série é estacionária quando se 
desenvolve no tempo aleatoriamente ao redor de uma média constante, retratando algum equilíbrio estável. Um processo é ergódico quando se podem estimar características de interesse (média, autocovariância, etc) a partir de uma única trajetória do processo.

Portanto, para modelar a volatilidade dos retornos gerados pelas commodities, calcularam-se os retornos compostos continuamente ou $\log$-retorno por $r_{t}=\ln \left(\mathrm{P}_{\mathrm{t}}\right)-\ln \left(\mathrm{P}_{\mathrm{t}-1}\right)$.

O software utilizado para estimar a regressão dos dados e dos modelos de análise foi EVIEWS 5.0, da Quantitative Micro Software.

\section{Resultados e discussão}

A Figura 1 ilustra o comportamento das séries de preços e retornos da soja, café, milho e boi gordo, no período de análise.

(a) Séries de Preços e Retornos da Soja
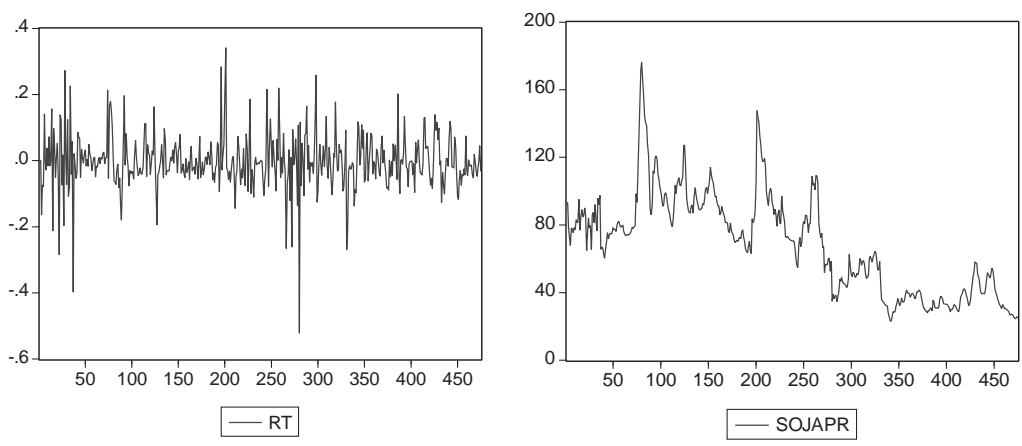
(b) Séries de Preços e Retornos do Café
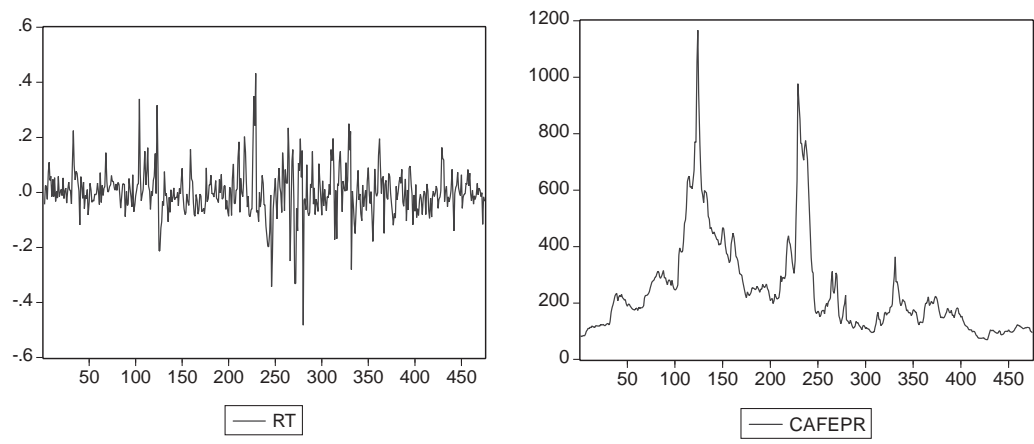

(c) Séries de Preços e Retornos do Milho
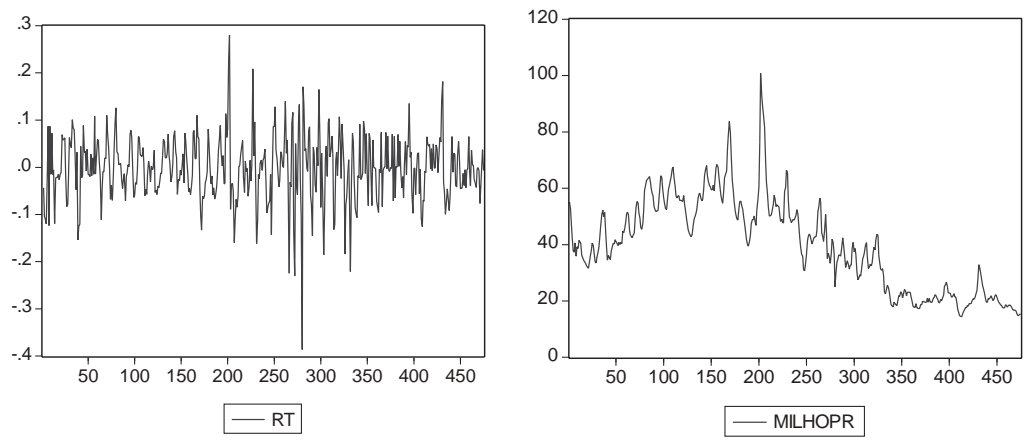
(d) Séries de Preços e Retornos do Boi Gordo
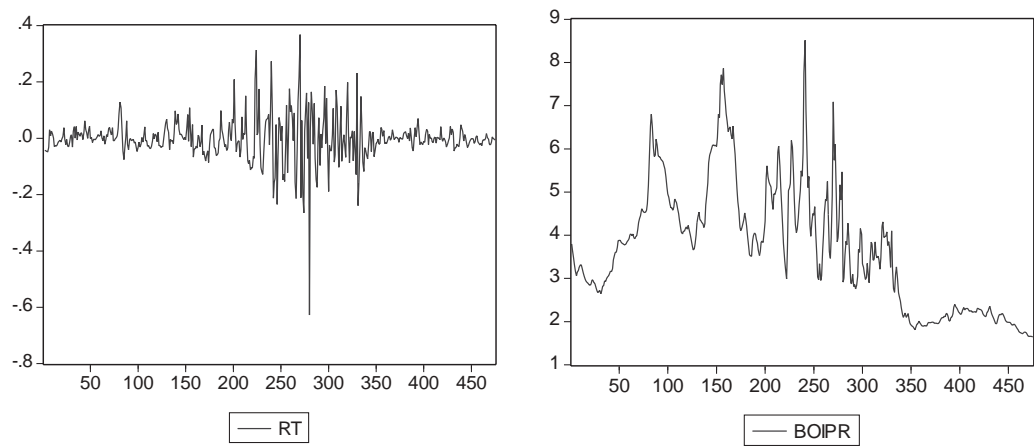

Figura 1 - Séries de preços e retornos da soja, café, milho e boi gordo (período de 1967 - 2006)

Fonte: Resultados da pesquisa.

A Tabela 1 mostra algumas estatísticas descritivas básicas para os retornos dos produtos agrícolas. O teste Jarque-Bera, de normalidade, é assintótico (grandes amostras) e calcula a assimetria e a curtose dos resíduos. A sua finalidade é testar a hipótese nula de que a amostra foi extraída de uma distribuição normal, em que o valor da assimetria é zero e o valor da curtose, três. Os resultados do teste mostram que os resíduos não apresentam distribuição normal.

Tabela 1 - Estatísticas descritivas

\begin{tabular}{ccccc}
\hline Estatísticas & Soja & Café & Milho & Boi Gordo \\
\hline Média & $-0,002775$ & 0,000245 & $-0,002709$ & $-0,001765$ \\
Mediana & $-0,008981$ & $-0,003115$ & $-0,005512$ & $-0,002998$ \\
Desvio-Padrão & 0,081614 & 0,087755 & 0,067399 & 0,074166 \\
Assimetria & $-0,357792$ & $-0,005621$ & $-0,327328$ & $-0,827300$ \\
Curtose & 9,060826 & 8,216849 & 6,136934 & 16,53287 \\
Teste Jarque-Bera & 735,6021 & 537,5088 & 202,8113 & 3671,058 \\
Probabilidade (JB) & 0,000000 & 0,000000 & 0,000000 & 0,000000 \\
\hline
\end{tabular}

Fonte: Resultados da pesquisa. 
A assimetria retrata a forma de distribuição dos dados, em que há um coeficiente igual a $-0,357792$ para a soja, ou seja, como a média é maior do que a mediana, tem-se uma assimetria à direita. A curtose de 9,060826 indica que a série de retornos apresenta uma distribuição leptocúrtica em relação à normal, o que indica, juntamente com as outras medidas descritivas e a representação gráfica do comportamento dos preços e dos retornos ao longo do período de análise, que a série do produto soja exibe sinais de heterocedasticidade e de agrupamento de volatilidade.

A Tabela também mostra as estatísticas descritivas básicas para os retornos do café, milho e boi gordo. O teste Jarque-Bera, de normalidade, calculado para ambas as culturas, também prova que os resíduos não apresentam distribuição normal. O coeficiente de assimetria desses produtos foi diferente de zero, com presença de assimetria à direita ou positiva. A análise da curtose, que é uma medida do pico ou do achatamento da distribuição, explica que os dados estão agrupados no centro juntamente com algumas observações nos extremos das caudas, representando séries de retornos com distribuição leptocúrtica ou aguda em relação à normal. Logo, as séries dos produtos café, milho e boi gordo também exibem sinais de heterocedasticidade e de agrupamento de volatilidade.

O primeiro procedimento foi ajustar um modelo ARMA $(p, q)$ à série de retornos, para eliminar a correlação serial entre as observações. A Tabela 2 apresenta as funções de autocorrelações (FAC) e funções de autocorrelações parciais (FACP), de forma que uma análise da FAC e da FACP do correlograma dos retornos e dos retornos quadráticos indica um modelo AR (1), MA (1) e MA (2) para a cultura da soja.

O ajustamento do modelo para correção da correlação foi feito ao eliminar vários coeficientes não-significativos a $10 \%$ de significância. A análise dos resíduos do modelo corrigido forneceu $\mathrm{Q}(20)=27,872$, com valor de probabilidade igual a 0,112 , o que mostra a eliminação da correlação serial da série de retornos mensais da soja. 
Tabela 2 - Estimativas dos coeficientes de autocorrelação e autocorrelação parcial para retornos e retornos quadráticos

\begin{tabular}{|c|c|c|c|c|c|c|c|c|c|}
\hline \multicolumn{5}{|c|}{ Retornos - Soja } & \multicolumn{5}{|c|}{ Retornos Quadráticos - Soja } \\
\hline $\mathbf{K}$ & C & FACP & Q-Stat & b & $\mathbf{K}$ & FAC & FACP & Q-Stat & rob \\
\hline 1 & & & & & & & & & \\
\hline 2 & & & & & 2 & & & & \\
\hline 3 & & & & & & & & & \\
\hline T & & & & & & & & & \\
\hline$J$ & & & & & J & & & & \\
\hline 6 & & & & & 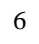 & & & & \\
\hline 7 & & & & & & & & & \\
\hline 8 & & & & & & & & & \\
\hline 9 & & & & & 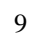 & & & & \\
\hline & & & & & & & & & \\
\hline \multicolumn{5}{|c|}{ Retornos - Café } & \multicolumn{5}{|c|}{ Retornos Quadráticos - Café } \\
\hline $\mathbf{K}$ & FAC & FACP & Q-Sta & & 3 & & & & \\
\hline 1 & & & & & & & & & \\
\hline 2 & & & & & & & & & \\
\hline 3 & & & & & & & & & \\
\hline 4 & -0.070 & & & 0.000 & 4 & 28 & & & ח \\
\hline & & -0.013 & 63.035 & & 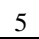 & & & & \\
\hline \multicolumn{5}{|c|}{ Retornos - Milho } & \multicolumn{5}{|c|}{ Retornos Quadráticos - Milho } \\
\hline N & & & & & 18 & & & & \\
\hline 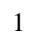 & & & & & & & & & \\
\hline 2 & & & & & & & & & \\
\hline J & & & & & 3 & & & & \\
\hline 4 & & & & & 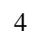 & & & & 000 \\
\hline & -0.168 & & & & & 0.070 & & & 0.000 \\
\hline \multicolumn{5}{|c|}{ Retornos - Boi Gordo } & \multicolumn{5}{|c|}{ Retornos Quadráticos - Boi Gordo } \\
\hline - & & & & & & $\mathrm{C}$ & & & rob \\
\hline 1 & & & & & & & & & 0.003 \\
\hline 2 & -0.035 & & & & 2 & & & & 0.001 \\
\hline 3 & -0.103 & -0.085 & 23.246 & 0.000 & 3 & 0.138 & 0.114 & 24.256 & 0.000 \\
\hline 4 & -0.135 & & & 0.000 & 4 & 0.120 & 0.083 & & 0.000 \\
\hline 5 & -0.182 & -0.154 & 47.947 & 0.000 & 5 & 0.080 & 0.035 & 34.236 & 0.000 \\
\hline
\end{tabular}

$\mathrm{k}=$ defasagens; FAC $=$ coeficientes de autocorrelação; FACP $=$ coeficientes de autocorrelação parcial; Q-Stat = teste de significância das autocorrelações; Prob = probabilidade do teste de significância.

Fonte: Resultados da pesquisa. 
Então, a Tabela 2 apresenta as funções de autocorrelações (FAC) e funções de autocorrelações parciais (FACP) de todos os produtos agropecuários, de forma que uma análise desses coeficientes tende a uma indicação de ajustamento de modelo AR (1), AR (3) e AR (9) para o café e AR (1), AR (2) e MA (1) para a cultura do milho e boi gordo. Após o ajustamento do modelo, a análise do correlograma dos resíduos forneceu $\mathrm{Q}(30)=36,219, \mathrm{Q}(11)=13,804$ e $\mathrm{Q}(12)=15,395$, com valores de probabilidade iguais a 0,201,0,244 e 0,221, o que mostra a eliminação da correlação serial da série de retornos mensais do café, milho e boi gordo.

Para confirmar a existência de volatilidade da série de retorno com padrão ARCH, realizou-se o teste do tipo multiplicador de Lagrange (Teste LM), proposto por Engle (1982), nos resíduos dos modelos AR e MA ajustados na regressão dos retornos. A Tabela 3 mostra os valores de probabilidade do teste e os resultados do teste LM, que indicam a presença do efeito ARCH na série de retornos de todos os produtos agrícolas. As estatísticas F e LM rejeitam a hipótese nula de que não há presença do efeito $\mathrm{ARCH}$ na série de retorno, ou seja, permitem que se anule a presença da homocedasticidade nos resíduos dos retornos.

Tabela 3 - Teste ARCH, de Engle (1982), dos retornos de soja, café, milho e boi gordo

\begin{tabular}{cc|cc|cc|cc}
\hline \multicolumn{2}{c|}{ Resíduos/AR (1), MA } & \multicolumn{2}{|c|}{ Resíduos/AR (1), AR } & \multicolumn{2}{c|}{ Resíduos/ AR (1), AR } & \multicolumn{2}{c}{ Resíduos/ AR (1), AR (2) } \\
\multicolumn{2}{c|}{ (1) e MA (2) - Soja } & \multicolumn{2}{c|}{ (3) e AR (9) - Café } & (2) e MA (1) - Milho & \multicolumn{2}{c|}{ e MA (1) - Boi } \\
\hline Lag & Probabilidade & Lag & Probabilidade & Lag & Probabilidade & Lag & Probabilidade \\
1 & 0,012504 & 1 & 0,001404 & 1 & 0,000000 & 1 & 0,000099 \\
5 & 0,163964 & 5 & 0,001656 & 5 & 0,000000 & 5 & 0,001638 \\
10 & 0,055765 & 10 & 0,002235 & 10 & 0,000000 & 10 & 0,000003 \\
20 & 0,063278 & 20 & 0,004978 & 20 & 0,000000 & 20 & 0,000964 \\
\hline
\end{tabular}

Fonte: Resultados da pesquisa.

A Tabela 3 também expõe o valor de probabilidade do teste do multiplicador, de Lagrange, para as respectivas defasagens e indica a presença do efeito ARCH nas séries de retornos. As estatísticas F também rejeitam a hipótese nula de que não há presença do efeito $\mathrm{ARCH}$ nas séries de retornos. 
Conforme Tabela 4, no ajustamento da série do modelo à média condicional, identificou-se que o melhor modelo para a cultura da soja foi um AR (1) para o modelo da classe $\mathrm{ARCH}$ (1), com parâmetros estatisticamente significativos a $10 \%$ de significância. Pela posterior análise dos correlogramas dos resíduos padronizados e dos quadrados dos resíduos padronizados, concluiu-se que não existe heterocedasticidade condicional nos resíduos do modelo ajustado. 
Tabela 4 - Estimação do Modelo ARCH para a série de retornos da soja, café, milho e boi gordo

\begin{tabular}{ccccc}
\hline \multicolumn{5}{c}{ Método: ML - ARCH (Marquardt) - Distribuição Normal } \\
\hline \multicolumn{5}{c}{ Série de Retornos da Soja } \\
\hline Coeficiente & Erro-Padrão & Estatística z & Probabilidade \\
AR (1) & 0.258091 & 0.052556 & 4.910782 & 0.0000 \\
\hline \multicolumn{5}{c}{ Equação de Variância } \\
\hline C & 0.004179 & 0.000222 & 18.84158 & 0.0000 \\
RESID $(-1)^{\wedge} 2$ & 0.484314 & 0.059578 & 8.129063 & 0.0000 \\
\hline
\end{tabular}

Série de Retornos do Café

\begin{tabular}{ccccc}
\hline & Coeficiente & Erro-Padrão & Estatística z & Probabilidade \\
AR (1) & 0.250042 & 0.031359 & 7.973586 & 0.0000 \\
\hline \multicolumn{5}{c}{ Equação de Variância } \\
\hline C & 0.004580 & 0.000232 & 19.74567 & 0.0000 \\
RESID $(-1)^{\wedge} 2$ & 0.421842 & 0.057064 & 7.392417 & 0.0000 \\
\hline
\end{tabular}

Série de Retornos do Milho

\begin{tabular}{|c|c|c|c|c|}
\hline & Coeficiente & Erro-Padrão & Estatística $\mathrm{z}$ & Probabilidade \\
\hline AR (1) & 1.345828 & 0.051860 & 25.95097 & 0.0000 \\
\hline $\operatorname{AR}(2)$ & -0.471952 & 0.043726 & -10.79349 & 0.0000 \\
\hline MA (1) & -0.931072 & 0.029345 & -31.72808 & 0.0000 \\
\hline \multicolumn{5}{|c|}{ Equação de Variância } \\
\hline $\mathrm{C}$ & 0.002415 & 0.000164 & 14.67888 & 0.0000 \\
\hline $\operatorname{RESID}(-1)^{\wedge} 2$ & 0.356973 & 0.056419 & 6.327153 & 0.0000 \\
\hline \multicolumn{5}{|c|}{ Série de Retornos do Boi Gordo } \\
\hline & Coeficiente & Erro-Padrão & Estatística $\mathrm{z}$ & Probabilidade \\
\hline AR (1) & 0.426575 & 0.022455 & 18.99704 & 0.0000 \\
\hline \multicolumn{5}{|c|}{ Equação de Variância } \\
\hline $\mathrm{C}$ & 0.000268 & $3.24 \mathrm{E}-05$ & 8.292173 & 0.0000 \\
\hline $\operatorname{RESID}(-1)^{\wedge} 2$ & 0.780723 & 0.091540 & 8.528801 & 0.0000 \\
\hline $\operatorname{RESID}(-2)^{\wedge} 2$ & 0.331537 & 0.060857 & 5.447829 & 0.0000 \\
\hline $\operatorname{RESID}(-3)^{\wedge} 2$ & 0.293145 & 0.045660 & 6.420103 & 0.0000 \\
\hline
\end{tabular}

Fonte: Resultados da pesquisa. 
Já no ajustamento da série do modelo para a média condicional, constatouse que os melhores modelos para os demais produtos seriam AR (1) para o café e AR (1), AR (2) e MA (1) para o milho, dado o modelo da classe ARCH (1) com parâmetros estatisticamente significativos a $1 \%$. Para o produto boi gordo, o modelo foi ajustado a partir de um AR (1) para os modelos ARCH (3), identificando-se também parâmetros significativos a $1 \%$.

Esses resultados estão de acordo com os do trabalho desenvolvido por Silva, Sáfadi e Castro Júnior (2005), segundo os quais os melhores modelos para a série de retornos da soja são um AR (1), para os modelos GARCH $(1,1)$, TARCH $(1,1)$ e TARCH $(1,2)$ na variância, e um AR (2) incompleto, para o modelo EGARCH $(1,1)$. Para a série de retornos do café, o melhor modelo também foi um AR (1) para todos os modelos da classe ARCH na variância.

Em seguida, pela análise dos correlogramas dos resíduos padronizados e dos quadrados dos resíduos padronizados, concluiu-se que não existe heterocedasticidade condicional nos resíduos dos modelos ajustados.

De acordo com a Tabela 5, a estimação do $\operatorname{GARCH}(1,1)$ permitiu captar a dinâmica da volatilidade na série de retorno dos produtos em análise. As condições observadas para que a variância do processo seja positiva e fracamente estacionária é que os parâmetros da regressão sejam positivos e maiores que zero. Assim, na equação de regressão, o segundo parâmetro representado pelo ARCH constitui o coeficiente de reação da volatilidade e o terceiro parâmetro $(\mathrm{GARCH})$, o coeficiente de persistência da volatilidade ou o risco na série de retorno. 
Tabela 5 - Estimação do Modelo GARCH $(1,1)$ para a série de retornos da soja, café, milho e boi gordo

Método: ML - ARCH (Marquardt) - Distribuição Normal

Série de Retornos da Soja

\begin{tabular}{ccccc}
\hline & $\begin{array}{c}\text { Coeficiente } \\
\text { AR (1) }\end{array}$ & $\begin{array}{c}\text { Erro-Padrão } \\
0.308560\end{array}$ & $\begin{array}{c}\text { Estatística z } \\
\text { Probabilidade } \\
\text { Equação de Variância }\end{array}$ & $\begin{array}{c}\text { P.628351 } \\
0.0000\end{array}$ \\
\hline C & 0.001045 & 0.000186 & 5.625582 & 0.0000 \\
RESID (-1)^2 & 0.343735 & 0.046595 & 7.377149 & 0.0000 \\
GARCH (-1) & 0.559020 & 0.052455 & 10.65705 & 0.0000 \\
\hline
\end{tabular}

Série de Retornos do Café

\begin{tabular}{ccccc}
\hline & $\begin{array}{c}\text { Coeficiente } \\
\text { AR (1) }\end{array}$ & $\begin{array}{c}\text { Erro-Padrão } \\
0.305815\end{array}$ & $\begin{array}{c}\text { Estatística z } \\
\text { Probabilidade } \\
\text { Equação de Variância }\end{array}$ & $\begin{array}{c}\text { P.920756 } \\
0.0000\end{array}$ \\
\hline C & 0.000303 & $8.47 \mathrm{E}-05$ & 3.578524 & 0.0003 \\
RESID (-1)^2 & 0.122705 & 0.026044 & 4.711499 & 0.0000 \\
GARCH (-1) & 0.838994 & 0.031631 & 26.52430 & 0.0000 \\
\hline
\end{tabular}

Série de Retornos do Milho

\begin{tabular}{ccccc}
\hline & Coeficiente & Erro-Padrão & Estatística z & Probabilidade \\
AR (1) & 1.379848 & 0.055364 & 24.92323 & 0.0000 \\
AR (2) & -0.514513 & 0.045010 & -11.43100 & 0.0000 \\
MA (1) & -0.915032 & 0.033504 & -27.31122 & 0.0000 \\
\hline \multicolumn{5}{c}{ Equação de Variância } \\
\hline C & 0.000427 & 0.000139 & 3.078572 & 0.0021 \\
RESID (-1)^2 & 0.200418 & 0.056676 & 3.536186 & 0.0004 \\
GARCH (-1) & 0.688390 & 0.077081 & 8.930687 & 0.0000 \\
\hline
\end{tabular}

Série de Retornos do Boi Gordo

\begin{tabular}{ccccc}
\hline & $\begin{array}{c}\text { Coeficiente } \\
\text { AR (1) }\end{array}$ & $\begin{array}{c}\text { Erro-Padrão } \\
0.434098\end{array}$ & $\begin{array}{c}\text { Estatística z } \\
12.33411\end{array}$ & $\begin{array}{c}\text { Probabilidade } \\
0.0000\end{array}$ \\
\hline \multicolumn{5}{c}{ Equação de Variância } \\
\hline C & $2.17 \mathrm{E}-05$ & $9.43 \mathrm{E}-06$ & 2.302547 & 0.0213 \\
RESID (-1)^2 & 0.290125 & 0.044702 & 6.490145 & 0.0000 \\
GARCH (-1) & 0.753965 & 0.032134 & 23.46324 & 0.0000 \\
\hline
\end{tabular}

Fonte: Resultados da pesquisa. 
A soma dos coeficientes de reação (ARCH) com o coeficiente de persistência da volatilidade (GARCH) define se os riscos persistem na série de retornos. Portanto, observa-se que o somatório dos coeficientes na cultura da soja foi igual a 0,902755 , o que indica elevada persistência de choques na volatilidade dos retornos da soja.

Logo, um choque que gere declínio ou aumento do preço da soja pode implicar vários períodos de intensa instabilidade ou volatilidade no mercado de soja, gerando perdas consideráveis para o setor agrícola e para a economia nacional.

Ao analisar agora as demais culturas, a estimação do $\operatorname{GARCH}(1,1)$ também identificou a dinâmica da volatilidade na série de retorno. A soma dos coeficientes de reação $(\mathrm{ARCH})$ com o coeficiente de persistência da volatilidade $(\mathrm{GARCH})$ definiu valores da ordem de 0,961699 (café), 0,888808 (milho) e 1,044090 (boi gordo), o que indica, assim como a soja, intensa volatilidade dos retornos.

De acordo com o coeficiente de persistência, a série de retornos do boi gordo apresentou valor maior do que um, o que demonstra que os choques na volatilidade irão perdurar por um período extremamente longo e que a variância permanecerá elevada, não apresentando reversão à sua média histórica. Observam-se, para as séries do café e soja, valores próximos de um, ou seja, mais vagarosamente o choque sobre a volatilidade irá se dissipar e, portanto, maior será a demora do processo de reversão à média para a variância. O menor coeficiente encontrado foi o do milho, o que mostra que um choque inicial sobre a volatilidade irá provocar efeitos mais rápidos sobre o comportamento da série (menor período de tempo), em comparação com outras culturas analisadas.

Em estudo desenvolvido por Silva, Sáfadi e Castro Júnior (2005), verificouse o somatório dos coeficientes do modelo $\operatorname{GARCH}(1,1)$ igual a 0,956 e 0,88 para soja e café, respectivamente, o que mostra elevada persistência de choques sobre a volatilidade dos retornos desses produtos agrícolas. 
Assim, o somatório desses dois parâmetros indica que, se o valor for maior ou próximo de um, maior será o efeito ao longo do tempo de qualquer instabilidade no mercado agropecuário. Consequientemente, esse efeito, decorrente de constantes flutuações de preços e produção do mercado agrícola, tende à normalidade num maior espaço de tempo, tornando essas commodities altamente vulneráveis ao mercado de futuros.

O mercado agropecuário está sujeito a grandes variações de preços dos produtos ocasionadas por fatores naturais e econômicos, tais como dificuldades impostas ao planejamento da produção, instabilidade no abastecimento, em decorrência de perdas em períodos de intensa estiagem ou chuvas, e as flutuações nos índices de preços, em razão de queda ou intensidade da colheita, de oscilações das cotações nos mercados internacionais e da variabilidade cambial.

Especialmente no caso do milho, deve ser enfatizado que há necessidade de reter estoques suficientes para que seja possível regularizar a oferta doméstica em anos de redução da produção, garantindo a sustentação dos preços internos e diminuindo sua volatilidade. Além disso, a sustentação de preços é estratégica também para a criação de excedentes exportáveis e para a redução na instabilidade financeira dos produtores.

A sustentação de preços via utilização de novos instrumentos de comercialização, tais como os contratos de opção de venda de produtos agrícolas e o programa de escoamento da produção (PEP), pode ser uma tentativa de amenizar as incertezas para o produtor. Os contratos de opção de venda constituem um seguro contra a queda de preços. $\mathrm{O}$ produtor ou a cooperativa, ao comprar um contrato de opção de venda, paga um preço (chamado prêmio) e passa a ter o direito de vender sua produção a um valor preestabelecido (chamado preço de exercício), na data de vencimento do contrato (Conceição, 2002).

O programa de escoamento da produção tem a finalidade de garantir um preço de referência ao produtor e às cooperativas e, ao mesmo tempo, contribuir para o abastecimento interno. O preço de referência é definido 
pelo Governo Federal, com base no preço mínimo e no preço de exercício das opções. Este programa permite que a iniciativa privada adquira a produção no período de safra, garantindo ao produtor o preço mínimo.

\section{Conclusão}

As flutuações cíclicas e, ou, sazonais dos preços dos produtos agropecuários provocam instabilidade tanto na renda do produtor como nas despesas dos consumidores urbanos. Assim, é necessário o conhecimento do padrão de flutuação sazonal ou a volatilidade destes preços, para que possam ser implementadas políticas de estabilização dos preços dos produtos agropecuários ao longo do ano.

A análise empírica da volatilidade dos retornos de principais commodities agrícolas (soja, café, milho e boi gordo) retrata a importância desses produtos pelo caráter exportador ou como produto de suma importância na agricultura de subsistência do Brasil. Os mercados desses produtos são marcados por acentuadas flutuações de preços, o que indica significativas oscilações na rentabilidade dessas culturas e propicia aos agentes econômicos e especuladores mais informados maior lucratividade, dado o seu poder de previsões mais precisas sobre o comportamento do mercado.

Portanto, estimaram-se os modelo ARCH e GARCH para analisar a dinâmica da volatilidade na série de retornos da soja, café, milho e boi gordo, constatando, por meio do teste $\mathrm{ARCH}$, que as séries apresentaram heterocedasticidade condicional auto-regressiva em seus retornos, ou seja, choques positivos ou negativos nos preços dos produtos podem levar longos períodos de tempo para se normalizarem no mercado.

Ao observar o somatório dos coeficientes de reação (ARCH) com o coeficiente de persistência da volatilidade (GARCH), que define se os riscos persistem na série de retornos, constatam-se valores próximos ou maiores do que um, o que mostra que os choques na volatilidade irão 
perdurar por longo tempo. O coeficiente encontrado para a série de boi gordo foi maior que um, seguido, em ordem decrescente, pelos coeficientes das séries de café, soja e milho, o que indica o grau de instabilidade de produção e os rendimentos dessas commodities, respectivamente.

Verifica-se, portanto, que as informações sobre volatilidade são importantes para as previsões da variância condicional dos preços das commodities, em um horizonte indefinido de tempo. Ressalta-se o elevado risco de preço e de renda associado aos mercados destes produtos, o que pode proporcionar aos produtores e demais agentes econômicos grandes lucros em determinados períodos, mas também enormes prejuízos e mesmo a saída do mercado, em situações adversas.

Uma tentativa de superar ou amenizar esses fatos seria a utilização de novos instrumentos de comercialização pelos produtores por meio dos contratos de opção de venda de produtos agrícolas e do programa de escoamento da produção (PEP), com vistas em garantir a sustentação de preços de seus produtos.

Maior utilização de contratos em mercados futuros, em que operações de hedge possam ser efetuadas por produtores e por processadores da commodity, pode ser também uma tentativa de minimização do risco e das flutuações de preços, pois políticas governamentais de restrição ou criação de barreiras protecionistas no comércio interno ou externo nem sempre geram resultados positivos para os produtores e para o País. 


\section{Referências}

BOLLERSLEV, Tim. Generalized autoregressive conditional heteroscedasticity. Journal of Econometrics, v.31, n.3, p.307-327, 1986.

BOLLERSLEV, T.; ENGLE, R. F.; NELSON, D. B. ARCH models. In: ENGLE, R. F.; MACFADDEN, D. L. (Ed). Handbook of Econometrics. Amsterdam: North-Holland, 1994. v.4, cap. 49, p.29593038 .

CONCEIÇÃO, J. C. P. R. da. Contribuição dos novos instrumentos de comercialização para estabilização de preços e renda agrícolas. Texto para Discussão n 927. Brasília: IPEA, 2002.

ENGLE, R.F. Autoregressive conditional heteroskedasticity with estimates of the variance of U.K. inflation. Econométrica, v. 50, p. 987-1008, 1982.

FUNDAÇÃO GETÚLIO VARGAS (FGV). Disponível em http:// www.fgvdados.com.br. Acesso em: 15 nov. 2006.

GUJARATI, D. N. Econometria básica. São Paulo: Makron books, 2000.

LAMOUNIER, W. M. Comportamento dos preços no mercado "spot" de café do Brasil: análise nos domínios do tempo e da freqüência. Viçosa: UFV/DEA, 2001. 223p. (Tese de Doutorado)

MARQUARDT, D. W. "An Algorithm for Least Squares Estimation of Non Linear Parameters", Journal of the Society for Industrial and Applied Mathematics, v.11, p.431-441, 1963.

MORETTIN, P. A.; TOLOI, C. M. C. Análise de séries temporais. São Paulo: Edgard Blucher, 2004. 535 p. 
ORANJE, M. Competitividade das frutas brasileiras no comércio internacional. Viçosa: UFV/DER, 2003. 114p. (Dissertação de Mestrado)

PIMENTEL, E. A.; ALMEIDA, L.; SABBADINI, R. Comportamento recente das exportações agrícolas no Brasil: uma análise espacial no âmbito dos estados. In: Congresso brasileiro de economia e sociologia rural, 42, Cuiabá, 2004. Anais ..., Brasília : SOBER , 2004.

RODRIGUES, R. O papel do setor privado e os novos desafios do abastecimento nacional. Revista de Política Agrícola, v. 10, 2001.

SCHWAGER, J. D. Fundamental analysis. New York: John Wiley \& Sons, 1995.

SILVA, W. S. da ; SÁFADI, T.; CASTRO JÚNIOR, L. G. de. Uma análise empírica da volatilidade do retorno de commodities agrícolas utilizando modelos ARCH: os casos do café e da soja. Rio de Janeiro: Revista de Economia e Sociologia Rural, v.43, n.01, p.119 a 134. mar. 2005.

Abstract - The instability of the producers and investors income coming from price fluctuation is a problem whose characteristics and causes should be thoroughly investigated given the importance of the commodity in the national agribusiness and their losses in terms of profitability, jobs and exchange value to Brazil. Given that, it is used the class of autoregressive conditional heteroscedasticity models (ARCH and $\mathrm{GARCH}$ ) to characterize and analyze the volatility of the time series of monthly returns of soy, coffee, corn and fat ox. This analysis shows that these products are marked by having high price fluctuations, in which positive or negative shocks generate impacts in the long run. The sum of the volatility reaction and persistence coefficients showed values close or larger than one, indicating that the shocks up on volatility will last for a long period of time.

Key-words: price volatility, agricultural products, Brazil. 
REVISTA DE ECONOMIA E AGRONEGÓCIO, VOL.5, $N^{o} 3$ 